\title{
The Role of Radar Astronomy in Assessing and Mitigating the Asteroid Impact Hazard
}

\author{
Jean-Luc Margot ${ }^{1,2}$ and Jon D. Giorgini ${ }^{3}$ \\ ${ }^{1}$ Department of Physics and Astronomy, University of California, Los Angeles, CA 90095, USA \\ ${ }^{2}$ Department of Earth, Planetary, and Space Sciences, University of California, Los Angeles, \\ CA 90095, USA \\ email: jlm@astro.ucla.edu \\ ${ }^{3}$ Jet Propulsion Laboratory, Pasadena, CA 91109, USA \\ email: Jon.Giorgini@jpl.nasa.gov
}

\begin{abstract}
Radar instruments play a critical role in studies of Near-Earth Asteroids (NEAs) on two distinct levels: trajectory prediction and physical characterization.
\end{abstract}

Keywords. minor planets, asteroids, astrometry, radar astronomy

\section{Trajectory prediction}

Because of superb $\left(\sim 10^{-8}\right)$ fractional uncertainties in round-trip delay and Doppler measurements, which are orthogonal to plane-of-sky optical astrometry, radar measurements can decrease orbital parameter uncertainties by factors of $10^{3}-10^{5}$ and increase the interval over which an object's Earth close approaches can be reliably known at the three-sigma level of confidence by factors of 5-10 (Ostro and Giorgini 2004). The addition of radar data is particularly valuable for single-apparition objects and for objects that have been observed with an optical arc shorter than 10-20 years. The radar benefits can be secured for about 10-25\% of NEAs within five years of their detection (NASA 2007).

For NEAs having astrometry spanning two or more apparitions and future close planetary encounters, the primary source of uncertainty in trajectory prediction is usually the object-specific (and normally unmeasured) Yarkovsky effect. This has been demonstrated for the $\sim 1.2 \mathrm{~km}$ diameter object (29075) 1950 DA (Giorgini et al 2002, Busch et al.2007) and the $\sim 270 \mathrm{~m}$ diameter object (99942) Apophis (Giorgini et al 2008). Studies of Yarkovsky influences on near-Earth objects show that radar ranges on at least two apparitions improve the precision of the semi-major axis drift rate determinations on average by an order of magnitude compared to optical-only determinations (Nugent et al.2012). Therefore, radar data not only improve trajectory predictions but also improve our knowledge of uncertainties affecting the predictions, which allows us to better quantify the risk associated with NEAs.

\section{Physical Characterization}

Physical characterization of objects posing a significant risk will be paramount for any mitigation effort. For instance, effective mitigation efforts will require knowledge of multiplicity. Because 1 in 6 NEAs larger than $200 \mathrm{~m}$ is a binary (Margot et al 2002), radar observations to assess binarity will be essential. Optical observations of mutual events can reveal binarity in some geometries, but radar observations can detect satellites even if they do not cause detectable mutual events. Radar observations have uncovered the majority of known binary NEAs and all the known triple NEAs. Effective mitigation efforts 
Margot and Giorgini

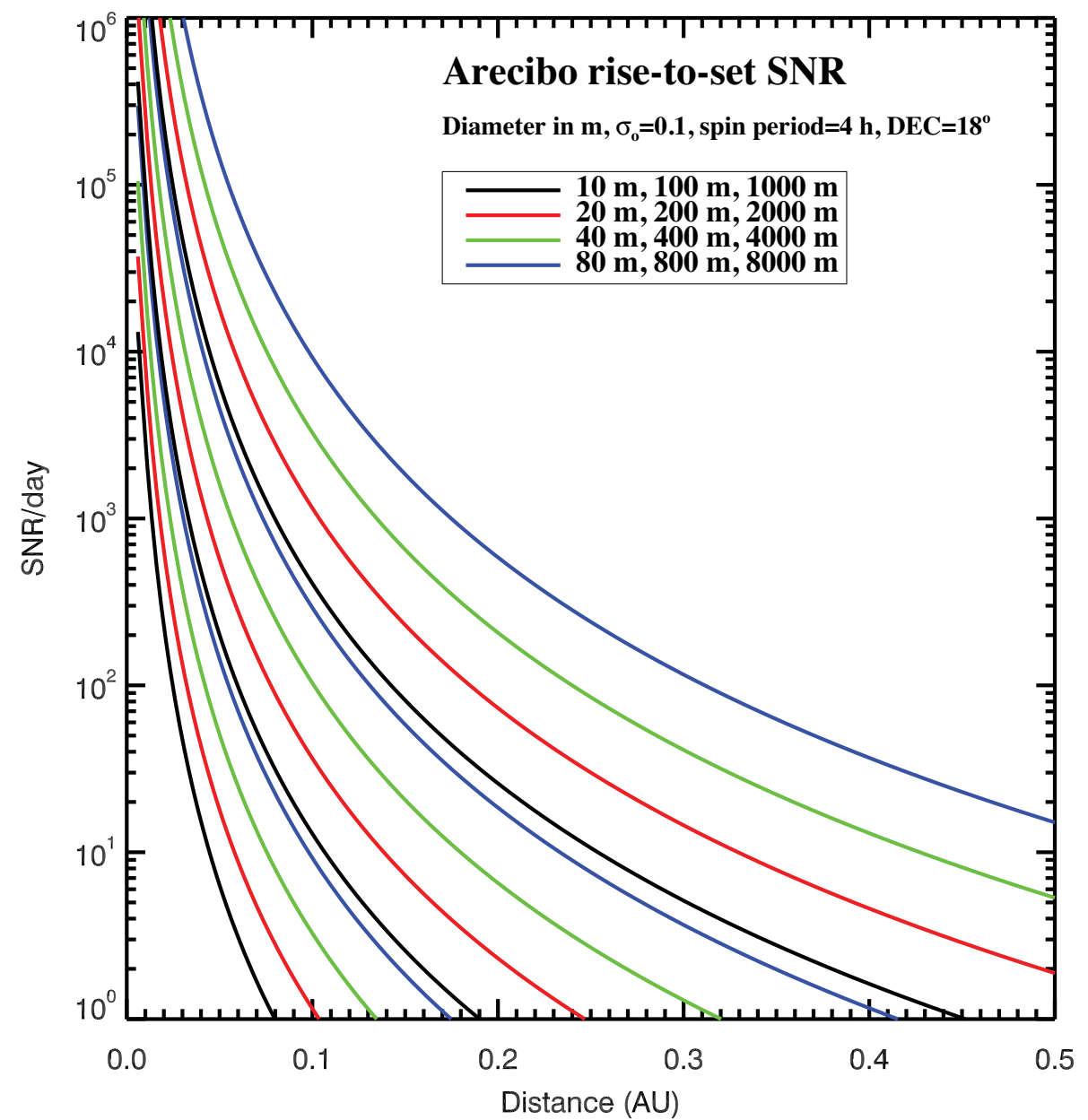

Figure 1. Arecibo signal-to-noise ratio (SNR) as a function of asteroid size and distance

would also likely be compromised without knowledge of the spin, shape, mass, density, and porosity. Radar observations provide the most realistic prospects, and sometimes the only realistic ground-based prospects, of securing estimates of all of these quantities. The combination of radar observations (Fig. 1) with observations at other wavelengths will provide the best possible ground-based characterization and will inform decisions about mitigation approaches.

\section{References}

Busch, M. W., et al. Icarus, 190:608, 2007.

Giorgini, J. D., et al. Icarus, 193:1, 2008.

Giorgini, J. D., et al. Science, 296:132, 2002.

Margot, J. L., et al. Science, 296:1445, 2002.

NASA, Near-Earth Object Survey and Deflection Analysis of Alternatives, 2007.

Nugent, C. R., Margot, J. L., Chesley, S. R., \& Vokrouhlický, D., AJ, 144:60, 2012.

Ostro, S. J. \& Giorgini, J. D. In Mitigation of Hazardous Comets and Asteroids. CUP, 2004. 